\title{
ON HOLOMORPHIC SOLUTIONS OF EQUATIONS OF KORTEWEG-DE VRIES TYPE
}

\author{
A. V. DOMRIN
}

\begin{abstract}
We show that, for any of the equations indicated in the title, every solution locally holomorphic in $x$ and $t$ admits global meromorphic continuation in $x$ for each $t$ with trivial monodromy at each pole. By way of application, we describe all possible envelops of meromorphy of local holomorphic solutions of the Boussinesq equation.
\end{abstract}

\section{$\S$ 1. IntroduCtion AND STATEMENT OF RESUlts}

Let $\mathcal{O}\left(x_{0}, t_{0}\right)$ be the set of all holomorphic function germs at a point $\left(x_{0}, t_{0}\right) \in \mathbb{C}^{2}$. Let us fix some integers $n, r \geq 2$. Following the fundamental papers [1] and [2, we use the term equations of Korteweg-de Vries type to denote evolution equations of the form

$$
L_{t}=[P, L],
$$

where the unknown functions are the coefficients $u_{0}, u_{1}, \ldots, u_{n-2} \in \mathcal{O}\left(x_{0}, t_{0}\right)$ of the linear differential operator $L=\partial_{x}^{n}+\sum_{j=0}^{n-2} u_{j}(x, t) \partial_{x}^{j}$ of order $n$, the operator $L_{t}$ is obtained from $L$ by the replacement of all coefficients by their partial $t$-derivatives (obviously, the order of $L_{t}$ does not exceed $\left.n-2\right)$, and the operator $P=\partial_{x}^{r}+\sum_{k=0}^{r-2} w_{k}(x, t) \partial_{x}^{k}$ is chosen in such a way that the order of the operator $[P, L]:=P L-L P$ does not exceed $n-2$ as well. This condition expresses the coefficients $w_{0}, w_{1}, \ldots, w_{r-2} \in \mathcal{O}\left(x_{0}, t_{0}\right)$ of $P$ as some polynomials of the germs $u_{0}, u_{1}, \ldots, u_{n-2}$ and their $x$-derivatives, this expression being unique up to $r-1$ arbitrary constants, which may depend on $t$. (See Proposition 2.3 in [1] or formula (1.4) below.) For example, the easy-to-verify identity

$$
\left[\partial_{x}^{3}+a \partial_{x}+b, \partial_{x}^{2}+c\right]=\left(3 c^{\prime}-2 a^{\prime}\right) \partial_{x}^{2}+\left(3 c^{\prime \prime}-a^{\prime \prime}-2 b^{\prime}\right) \partial_{x}+\left(c^{\prime \prime \prime}+a c^{\prime}-b^{\prime \prime}\right),
$$

where $a, b, c \in \mathcal{O}\left(x_{0}, t_{0}\right)$ and the prime stands for the $x$-derivative, readily implies that for $n=2$ and $r=3$ the condition ord $[P, L] \leq n-2$ is equivalent to the system of equations $w_{1}=\frac{3}{2} u+\gamma_{1}(t), w_{0}=\frac{3}{4} u^{\prime}+\gamma_{0}(t)$ with arbitrary germs $\gamma_{0}, \gamma_{1} \in \mathcal{O}\left(t_{0}\right)$, and then the evolution problem (1.1) is reduced to the Korteweg-de Vries equation for the function $u=u_{0}$,

$$
\frac{\partial u}{\partial t}=\frac{1}{4} \frac{\partial^{3} u}{\partial x^{3}}+\left(\frac{3}{2} u+\gamma_{1}(t)\right) \frac{\partial u}{\partial x} ;
$$

for $n=3$ and $r=2$, the same condition is equivalent to the equation $w_{0}=\frac{2}{3} u_{1}+\gamma_{0}(t)$ with an arbitrary germ $\gamma_{0} \in \mathcal{O}\left(t_{0}\right)$, and then the evolution problem (1.1) is reduced (for an arbitrary choice of $\left.\gamma_{0}(t)\right)$ to the Boussinesq system

$$
\frac{\partial u_{0}}{\partial t}=\frac{\partial^{2} u_{0}}{\partial x^{2}}-\frac{2}{3} \frac{\partial^{3} u_{1}}{\partial x^{3}}-\frac{2}{3} u_{1} \frac{\partial u_{1}}{\partial x}, \quad \frac{\partial u_{1}}{\partial t}=2 \frac{\partial u_{0}}{\partial x}-\frac{\partial^{2} u_{1}}{\partial x^{2}}
$$

2010 Mathematics Subject Classification. Primary 35Q53; Secondary 30B40.

Key words and phrases. Soliton equation, holomorphic solution, analytic continuation.

Supported by RFBR grants nos. 11-01-12033-ofi-m, 11-01-00495-a-2011, and 10-01-00178-a. 
In the general case, all operators of the form $P=\partial_{x}^{r}+\sum_{k=0}^{r-2} w_{k}(x, t) \partial_{x}^{k}$ satisfying the condition $\operatorname{ord}[P, L] \leq n-2$ are given by the formula

$$
P=L_{+}^{r / n}+\sum_{k=0}^{r-2} \gamma_{k}(t) L_{+}^{k / n}
$$

where $\gamma_{0}, \ldots, \gamma_{r-2} \in \mathcal{O}\left(t_{0}\right)$ are arbitrary germs and $L_{+}^{k / n}$ with integer $k \geq 2$ is the unique operator of the form $\partial_{x}^{k}+\sum_{l=0}^{k-2} W_{l}\left(u_{0}, \ldots, u_{n-2}\right) \partial_{x}^{l}$ whose commutator with $L$ is of order $\leq n-2$ and whose coefficients $W_{l}$ are homogeneous polynomials of weight $k-l$ of the functions $u_{0}, u_{1}, \ldots, u_{n-2}$ and their $x$-derivatives, each monomial $u_{j}^{(p)}$ being assigned the weight $n-j+p$. (Furthermore, we assume by definition that $L_{+}^{0 / n}$ is the identity operator and $L_{+}^{1 / n}=\partial_{x}$.) In the literature, one usually assumes that all germs $\gamma_{k}(t)$ are constant (as in [1, Eq. (2.3)]) or even zero (as in [2, Proposition 4.3]), although most of the results remain true for any $\gamma_{k}(t)$. We follow this tradition as well; i.e., we assume that $\gamma_{k}(t) \equiv \gamma_{k}=$ const in (1.4). In this case, the evolution problem (1.1) is a system of $n-1$ partial differential equations of the form

$$
\frac{\partial u_{j}}{\partial t}=F_{j}\left(u_{0}, u_{1}, \ldots, u_{n-2}\right), \quad j=0,1, \ldots, n-2,
$$

where $F_{0}, F_{1}, \ldots, F_{n-2}$ are some polynomials of the functions $u_{0}, u_{1}, \ldots, u_{n-2}$ and their $x$-derivatives. Note that formula (1.4) for $P$ will be used in our paper only once (in the proof of Lemma 3), while the explicit form of system (1.5) equivalent to Eq. (1.1) is not needed altogether except for the special cases (1.2) and (1.3) written out above.

Our aim is to study the analytic continuation of locally defined holomorphic solutions of Eq. (1.1), or, equivalently, of system (1.5). Here a solution is understood as the tuple $u_{0}(x, t), u_{1}(x, t), \ldots, u_{n-2}(x, t)$ of all coefficients of $L$. The main result is as follows.

Theorem. Let $n, r \geq 2$ be integers such that $r$ is not divisible by $n$. Let $u_{0}, u_{1}, \ldots, u_{n-2} \in$ $\mathcal{O}\left(x_{0}, t_{0}\right)$ be a tuple of germs that, in a neighborhood of a given point $\left(x_{0}, t_{0}\right) \in \mathbb{C}^{2}$, satisfies system (1.5) equivalent to Eq. (1.1) for some operator $P$ of the form (1.4) with constant coefficients $\gamma_{k}(t) \equiv \gamma_{k}=$ const, $k=0,1, \ldots, r-2$. Then the following claims hold.

(A) All germs $u_{0}\left(x, t_{0}\right), u_{1}\left(x, t_{0}\right), \ldots, u_{n-2}\left(x, t_{0}\right) \in \mathcal{O}\left(x_{0}\right)$ admit analytic continuation to functions $\widetilde{u}_{0}(x), \widetilde{u}_{1}(x), \ldots, \widetilde{u}_{n-2}(x)$ meromorphic on the entire plane $\mathbb{C}_{x}^{1}$ of the complex variable $x$.

(B) The operator $\widetilde{L}:=\partial_{x}^{n}+\sum_{j=0}^{n-2} \widetilde{u}_{j}(x) \partial_{x}^{j}$ has the following trivial monodromy property at all poles of its coefficients: for each $\lambda \in \mathbb{C}$, the ordinary differential equation $\widetilde{L} \varphi=\lambda \varphi$ has a fundamental solution system meromorphic on the entire plane $\mathbb{C}_{x}^{1}$ of the variable $x$. In other words, every solution $\varphi \in \mathcal{O}\left(x_{0}\right)$ of the equation $\widetilde{L} \varphi=\lambda \varphi$ admits analytic continuation to a meromorphic function on $\mathbb{C}_{x}^{1}$.

(C) All poles of each of the functions $\widetilde{u}_{j}(x), j=0,1, \ldots, n-2$, have order $\leq n-j$.

(D) Every solution $u_{0}, u_{1}, \ldots, u_{n-2} \in \mathcal{O}(D)$ of system (1.5) in an arbitrary bidisk $D:=\left\{(x, t) \in \mathbb{C}^{2}:\left|x-x_{0}\right|<\delta_{1},\left|t-t_{0}\right|<\delta_{2}\right\}$ can be analytically continued to a tuple of meromorphic functions in the strip $S:=\left\{(x, t) \in \mathbb{C}^{2}:\left|t-t_{0}\right|<\delta_{2}\right\}$ and in general cannot be analytically continued further across the boundary of $S$ at any point.

(E) However, all generic solutions $u_{0}, u_{1}, \ldots, u_{n-2} \in \mathcal{O}\left(x_{0}, t_{0}\right)$ (see Section $3(\mathrm{E})$ for the precise definition) admit analytic continuation to a tuple of meromorphic functions on the entire space $\mathbb{C}^{2}$ of the variables $x, t$. 
Corollary. Let $D:=\left\{(x, t) \in \mathbb{C}^{2}:\left|x-x_{0}\right|<\delta_{1},\left|t-t_{0}\right|<\delta_{2}\right\}$ be an arbitrary bidisk. Assume that a function $u \in \mathcal{O}(D)$ satisfies the Boussinesq equation

$$
\frac{\partial^{2} u}{\partial t^{2}}=a \frac{\partial^{4} u}{\partial x^{4}}+b\left(\frac{\partial u}{\partial x}\right)^{2}+b u \frac{\partial^{2} u}{\partial x^{2}}
$$

in $D$ for some $a, b \in \mathbb{C} \backslash\{0\}$. Then $u(x, t)$ admits analytic continuation to a meromorphic function in the strip $S:=\left\{(x, t) \in \mathbb{C}^{2}:\left|t-t_{0}\right|<\delta_{2}\right\}$ and in general cannot be analytically continued further across the boundary of $S$ at any point. More generally, the envelope of meromorphy of any locally defined solution $u \in \mathcal{O}\left(x_{0}, t_{0}\right)$ of Eq. (1.6) has the form $\mathbb{C}_{x}^{1} \times X$ for some domain $X$ over $\mathbb{C}_{t}^{1}$ (i.e., for an arcwise connected complex manifold $X$ admitting a locally invertible holomorphic mapping $\pi: X \rightarrow G$ onto some domain $G \subset \mathbb{C}_{t}^{1} ;$ e.g., see [3, Section II.7.22]), and conversely, for each such $X$ and every point $\left(x_{0}, t_{0}\right) \in \mathbb{C}^{1} \times G$ there exists a solution $u \in \mathcal{O}\left(x_{0}, t_{0}\right)$ of Eq. (1.6) whose envelope of meromorphy coincides with $\mathbb{C}_{x}^{1} \times X$.

Thus, the envelope of meromorphy of a local holomorphic solution of the Boussinesq equation always fills the entire plane $\mathbb{C}^{1}$ in the $x$-direction and can be completely arbitrary in the $t$-direction. Similar assertions hold for all germs $u_{0}, u_{1}, \ldots, u_{n-2}$ in the assumptions of the theorem, but we do not prove this in the present paper. All claims of the corollary are also true for the Korteweg-de Vries equation (1.2) with $\gamma_{1}(t) \equiv \gamma_{1}=$ const and for many other soliton equations. (See Remark 6 at the end of the paper.) However, this result was essentially obtained by a different method in [4] (although it was not stated there explicitly).

The proof of the theorem is given in Section 3 (following some preliminary work in Section 2), and the Corollary is proved in Section 4. Let us close the introduction with some remarks concerning terminology, literature, and known results.

Remark 1. Equations of the form (1.1) for general $n$ and $r$ apparently occurred for the first time in Krichever's paper [5] and Gelfand-Dikii's paper [6] in 1976. The term equations of Korteweg-de Vries type, motivated by the special case (1.2), stuck after the above-mentioned papers [1] by Drinfel'd and Sokolov and 2] by Segal and Wilson, published in the first half of the 1980s. The family of equations of the form (1.1) with fixed $n$ and with all possible operators $P$ of the form (1.4) (with constant $\gamma_{k}(t)$ and often even with $\gamma_{k}(t) \equiv 0$ for all $\left.k=0,1,2, \ldots\right)$ is also called the $n$th generalized $K d V$ hierarchy or the $n$th Gelfand-Dikii hierarchy (e.g., see Definition 1.4.4 and Remark 1.8.15 in [7, where further bibliographical references can be found). The term scalar Lax equations is used as well. (See Section 2 in [1] or Section 2.1 of the survey [8.) Of the immense literature related in some or other way to equations of the form (1.1), we only mention the monographs [7] and [9] and the survey papers [10] and [11, almost completely dedicated to the subject. To the best of the author's knowledge, the problem on analytic continuation of arbitrary holomorphic solutions of equations of Korteweg-de Vries type has not been considered in the literature. Existing results for narrower classes of holomorphic solutions are discussed in the next remark.

Remark 2. An operator $L_{0}=\partial_{x}^{n}+\sum_{j=0}^{n-2} q_{j}(x) \partial_{x}^{j}$ with coefficients $q_{j} \in \mathcal{O}\left(x_{0}\right)$ is said to be finite-gap if there exists an operator $P_{0}=\partial_{x}^{m}+\sum_{k=0}^{m-2} p_{k}(x) \partial_{x}^{k}$ with coefficients $p_{k} \in \mathcal{O}\left(x_{0}\right)$ such that $m$ and $n$ are coprime and $\left[P_{0}, L_{0}\right] \equiv 0$ in a neighborhood of $x_{0} \in \mathbb{C}$. It is well known (see the original paper 12 or the survey [8]) that in this case all germs $q_{j}$ and $p_{k}$ extend to be meromorphic functions on $\mathbb{C}_{x}^{1}$ and that the Cauchy problem for any equation of the form (1.1) satisfying the assumptions of the theorem with the initial condition $L=L_{0}$ for $t=t_{0}$ has a unique solution $L$ in a neighborhood of the point $\left(x_{0}, t_{0}\right) \in \mathbb{C}^{2}$; moreover, this solution is meromorphic on the entire $\mathbb{C}^{2}$, and the identity 
$\left[P_{0}, L\right] \equiv 0$ is preserved for all $(x, t) \in \mathbb{C}^{2}$. Such solutions are also said to be finitegap (or algebraic-geometric). They form one of the best-studied classes of solutions for equations of Korteweg-de Vries type, and there is an immense literature dealing with these solutions, starting from Novikov's paper [13. The properties mentioned in claims (A), (C), and (E) of our theorem were rigorously proved by Segal and Wilson 2, Propositions 5.1 and 5.17, Theorem 6.10, and Remark 6.13] for all finite-gap solutions and even for the more general class of solutions with converging Baker-Akhiezer function. (This class, denoted by $\mathcal{C}^{(n)}$ in [2], was further studied in [14] and [15].) Weikard [16] was apparently the first to state the trivial monodromy property (B) explicitly for finite-gap solutions of general equations of Korteweg-de Vries type; he also established that this property is equivalent to the finite gap property for operators with rational coefficients holomorphic at infinity or with globally meromorphic periodic coefficients that have finite limits as $x \rightarrow \infty$ at both ends of the period strip. In this connection, note that the class of local holomorphic solutions dealt with in our theorem is by far not exhausted by finitegap solutions and even by solutions with converging Baker-Akhiezer function. This is already seen from claim (D) of the theorem. Moreover, one can modify an example in [4. Section 9(D)] to show that although the class of generic holomorphic solutions in claim (E) of the theorem contains the above-mentioned special solution classes, it is not exhausted by them as well.

\section{$\S 2$. Preparation for the proof of the Theorem}

All claims of the theorem will be proved by applying the results in [4 to soliton equations of parabolic type obtained from (1.1) by the Drinfel'd-Sokolov reduction [1]. This reduction, which is based on the replacement of an $n$ th-order linear differential equation by a system of $n$ first-order linear equations, was used in [1 to construct and study analogs of equations of Korteweg-de Vries type for arbitrary simple Lie algebras. This section presents some details of the reduction process, which are partly absent in [1] and the other papers cited above. For example, the statement of the following lemma, which represents Eq. (1.1) as a consistency condition for the auxiliary linear system (2.1), is essentially contained in [11, Theorem 5.7], but the proof given there is not suitable for our aims. Note also that the condition that $r$ is not divisible by $n$ in our theorem is not used in Section 2; its role will be revealed in Section 3(A) (see Remark 4).

Lemma 1. Let $n, r \geq 2$, and let operators $L=\partial_{x}^{n}+\sum_{j=0}^{n-2} u_{j}(x, t) \partial_{x}^{j}, P=\partial_{x}^{r}+$ $\sum_{k=0}^{r-2} w_{k}(x, t) \partial_{x}^{k}$ with coefficients $u_{j}, w_{k} \in \mathcal{O}\left(x_{0}, t_{0}\right)$ satisfy Eq. (1.1) in a neighborhood of the point $\left(x_{0}, t_{0}\right) \in \mathbb{C}^{2}$. Then for each $\lambda \in \mathbb{C}$ the system

$$
L \psi=\lambda \psi, \quad \psi_{t}=P \psi
$$

has $n$ linearly independent solutions $\psi_{1}, \ldots, \psi_{n} \in \mathcal{O}\left(x_{0}, t_{0}\right)$ such that the $n \times n$ matrix $\Psi(x, t, \lambda):=\left\|\partial_{x}^{i} \psi_{j}\right\|_{i, j=1}^{n}$ is nonsingular. Moreover, for each nonsingular matrix $\Psi_{0}(\lambda) \in$ $\mathrm{GL}(n, \mathbb{C})$ there exists a unique set of $n$ solutions $\psi_{1}, \ldots, \psi_{n} \in \mathcal{O}\left(x_{0}, t_{0}\right)$ of system (2.1) with the initial condition $\Psi\left(x_{0}, t_{0}, \lambda\right)=\Psi_{0}(\lambda)$.

Proof. Let germs $e_{1}, \ldots, e_{n} \in \mathcal{O}\left(x_{0}, t_{0}\right)$ form a fundamental solution system of the ordinary differential equation $L \psi=\lambda \psi$ for each $t$ close to $t_{0}$. Then the general solution $\psi \in \mathcal{O}\left(x_{0}, t_{0}\right)$ of this equation has the form $\psi(x, t)=\sum_{j=1}^{n} c_{j}(t) e_{j}(x, t)$ with arbitrary germs $c_{j} \in \mathcal{O}\left(t_{0}\right)$. By substituting this expression into the equation $\psi_{t}=P \psi$, we obtain the condition

$$
\sum_{j=1}^{n} c_{j}^{\prime} e_{j}+c_{j}\left(e_{j}^{\prime}-P e_{j}\right)=0
$$


on the coefficients $c_{1}, \ldots, c_{n}$. (Here and further, but only in this proof, the prime stands for the $t$-derivative.) The differentiation with respect to $t$ of the equation $L e_{j}=\lambda e_{j}$ gives the equation $L^{\prime} e_{j}+L e_{j}^{\prime}=\lambda e_{j}^{\prime}$, whence, by replacing $L^{\prime}$ with $[P, L-\lambda]$ according to (1.1), we obtain the relation $(L-\lambda)\left(e_{j}^{\prime}-P e_{j}\right)=0$. By expanding the solution $\varphi=e_{j}^{\prime}-P e_{j} \in \mathcal{O}\left(x_{0}, t_{0}\right)$ of the equation $L \varphi=\lambda \varphi$ in the basis $e_{1}, \ldots, e_{n}$, we write $e_{j}^{\prime}-P e_{j}=\sum_{k=1}^{n} d_{j k} e_{k}$ with some germs $d_{j k} \in \mathcal{O}\left(t_{0}\right)$ depending only on $t$. Then condition (2.2) acquires the form

$$
C^{\prime}(t)=D(t) C(t)
$$

where $C(t)=\left(c_{1}(t), \ldots, c_{n}(t)\right)^{T}$ is the column of unknown coefficients and $D(t)$ is a given $n \times n$ matrix with entries $D_{j k}(t):=-d_{k j}(t), 1 \leq j, k \leq n$. For each initial condition $C_{0} \in \mathbb{C}^{n}$, system (2.3) has a unique solution $C(t)$ with $C\left(t_{0}\right)=C_{0}$ holomorphic in a neighborhood of $t_{0}$, which is equivalent to the desired assertion.

One can derive the following linear system for the matrix $\Psi(x, t, \lambda)=\left\|\partial_{x}^{i} \psi_{j}\right\|_{i, j=1}^{n}$ from the auxiliary linear system (2.1):

$$
\Psi_{x}=(\Lambda(\lambda)+U(x, t)) \Psi, \quad \Psi_{t}=R(x, t, \lambda) \Psi,
$$

where all entries of the $n \times n$ matrices $\Lambda(\lambda)$ and $U(x, t)$ are zero except for $\Lambda_{i, i+1}(\lambda)=1$ for $1 \leq i \leq n-1, \Lambda_{n 1}(\lambda)=\lambda$, and $U_{n j}(x, t)=-u_{j-1}(x, t)$ for $1 \leq j \leq n-1$; all entries of the $n \times n$ matrix $R(x, t, \lambda)$ are polynomials in $\lambda$ with coefficients in $\mathcal{O}\left(x_{0}, t_{0}\right)$. The degrees of these polynomials do not exceed $A+1$, and the coefficient of $\lambda^{A+1}$ in $R(x, t, \lambda)$ is the matrix $\Lambda(1)^{B}$, where the integers $A \geq 0$ and $B \in\{0,1, \ldots, n-1\}$ are uniquely determined by the equation $r=A n+B$. (Here we have taken into account the fact that $\Lambda(\lambda)^{n}=\lambda I$ is a scalar multiple of the $n \times n$ identity matrix.) Indeed, the equation $L \psi=\lambda \psi$ for the function $\psi \in \mathcal{O}\left(x_{0}, t_{0}\right)$ is equivalent to the equation $\chi_{x}=(\Lambda(\lambda)+U(x, t)) \chi$ for the column $\chi=\left(\chi_{1}, \ldots, \chi_{n}\right)^{T}:=\left(\psi, \psi^{\prime}, \ldots, \psi^{(n-1)}\right)^{T}$, and the equation $\chi_{t}=R(x, t, \lambda) \chi$ can be obtained in this notation from the relation $\left(\chi_{j}\right)_{t}=$ $\psi_{t}^{(j-1)}=(P \psi)^{(j-1)}=\partial_{x}^{j-1}\left(\partial_{x}^{r}+\sum_{k=0}^{r-2} w_{k} \partial_{x}^{k}\right) \psi$ by replacing $\partial_{x}^{s} \psi$ by $\chi_{s-1}$ for $1 \leq s \leq n$ and by expressing all $\partial_{x}^{s} \psi$ for $s \geq n+1$ via $\chi_{1}, \ldots, \chi_{n}$ with the use of the formula $\partial_{x}^{n} \psi=-\sum_{j=0}^{n-2} u_{j} \partial_{x}^{j} \psi+\lambda \psi$, equivalent to the equation $L \psi=\lambda \psi$, and the formulas obtained from it by differentiation with respect to $x$ appropriately many times. By way of illustration, note that the second equation in system (2.4) has the form

$$
\Psi_{t}=\left(\begin{array}{cc}
w_{0}-u^{\prime} & \lambda-u+w_{1} \\
(\lambda-u)^{2}+\left(w_{1}-u^{\prime \prime}\right)(\lambda-u)+w_{0}^{\prime}+2{u^{\prime}}^{2} & -2 u^{\prime}(\lambda-u)+w_{1}^{\prime}+w_{0}
\end{array}\right) \Psi
$$

for the Korteweg-de Vries equation (1.2) and the form

$$
\Psi_{t}=\left(\begin{array}{ccc}
w_{0} & 0 & 1 \\
\lambda-u_{0}+w_{0}^{\prime} & w_{0}-u_{1} & 0 \\
w_{0}^{\prime \prime}-u_{0}^{\prime} & \lambda-u_{0}-u_{1}^{\prime}+2 w_{0}^{\prime} & w_{0}-u_{1}
\end{array}\right) \Psi
$$

for the Boussinesq system (1.3).

According to Ince [17, Section 5.21], the following classical statement concerning the decomposition of an $n$ th-order differential operator into a product of $n$ first-order operators is due to Frobenius and Floquet. There often arise various versions of this statement in modern papers on the theory of integrable systems (e.g., see the bibliographical references after formulas (5.55)-(5.58) in [11] or the lemma in the proof of Theorem 5 in [18]). The proof given below is a modification of the solution of Problem 62 in Part VII of Pólya-Szegö's book [19]. From now on, $W\left(h_{1}, \ldots, h_{j}\right)$ stands for the Wronskian of functions $h_{1}, \ldots, h_{j}$; i.e., $W\left(h_{1}, \ldots, h_{j}\right):=\operatorname{det}\left\|\partial_{x}^{k} h_{l}\right\|_{k, l=1}^{j}$. 
Lemma 2. Let $L=\partial_{x}^{n}+\sum_{j=0}^{n-1} q_{j}(x) \partial_{x}^{j}$ for some $q_{0}, \ldots, q_{n-1} \in \mathcal{O}\left(x_{0}\right)$, and suppose that $h_{1}, \ldots, h_{n} \in \mathcal{O}\left(x_{0}\right)$ form a fundamental solution system of the equation $L h=0$ such that all germs $W_{j}(x):=W\left(h_{1}, \ldots, h_{j}\right)(x), 1 \leq j \leq n$, are nonzero at the point $x_{0}$. Set $f_{j}(x):=W_{j}(x) / W_{j-1}(x)$ and $v_{j}(x):=f_{j}^{\prime}(x) / f_{j}(x)$ for $1 \leq j \leq n$, where $W_{0}(x): \equiv 1$ by convention. Then $L=\left(\partial_{x}-v_{n}(x)\right) \cdots\left(\partial_{x}-v_{1}(x)\right)$.

Proof. Note that for any germs $y_{1}, \ldots, y_{j} \in \mathcal{O}\left(x_{0}\right)$ such that $W\left(y_{1}, \ldots, y_{j}\right)\left(x_{0}\right) \neq 0$ there exists a unique operator of the form $K=\partial_{x}^{j}+\sum_{i=0}^{j-1} a_{i}(x) \partial_{x}^{i}, a_{j} \in \mathcal{O}\left(x_{0}\right)$, satisfying the condition $K y_{1}=\cdots=K y_{j}=0$. Namely, this operator is given by the formula $K y=$ $W\left(y_{1}, \ldots, y_{j}, y\right) / W\left(y_{1}, \ldots, y_{j}\right)$. (Indeed, the existence follows from this formula, and the uniqueness follows from the fact that the difference of two such operators has order $\leq j-1$ and vanishes on $j$ linearly independent functions.) Set $L_{j}:=\left(\partial_{x}-v_{j}(x)\right) \cdots\left(\partial_{x}-v_{1}(x)\right)$, $j=1, \ldots, n$. By the definition of $v_{1}$, the operator $L_{1}$ annihilates $h_{1}$ and hence is equal to $W\left(h_{1}, \cdot\right) / W\left(h_{1}\right)$ as was noted above, and then $L_{1} h_{2}=f_{2}$ by the definition of $f_{2}$. Induction over $j=1, \ldots, n$ shows in just the same way that $L_{j}$ annihilates the germs $h_{1}, \ldots, h_{j}$ and hence is equal to $W\left(h_{1}, \ldots, h_{j}, \cdot\right) / W\left(h_{1}, \ldots, h_{j}\right)$, and then (for $j<n$ ) we have $L_{j} h_{j+1}=f_{j+1}$. For $j=n$, the fact that $L_{n}$ annihilates $h_{1}, \ldots, h_{n}$, together with the uniqueness proved above, shows that $L_{n}=L$.

In the notation of Lemma 1 , choose a matrix $\Psi_{0}(0)=\Psi\left(x_{0}, t_{0}, 0\right)$ such that all of its principal minors be nonzero. Then for each $t$ close to $t_{0}$ we can apply Lemma 2 with $q_{j}(x)=u_{j}(x, t)$ and $h_{j}(x)=\psi_{j}(x, t, 0)$. We obtain germs $v_{1}, \ldots, v_{n} \in \mathcal{O}\left(x_{0}, t_{0}\right)$ such that

$$
\partial_{x}^{n}+\sum_{j=0}^{n-2} u_{j}(x, t) \partial_{x}^{j}=L=\left(\partial_{x}-v_{n}(x, t)\right) \cdots\left(\partial_{x}-v_{1}(x, t)\right)
$$

in a neighborhood of $\left(x_{0}, t_{0}\right)$. By matching the coefficients of $\partial_{x}^{n-1}$, we see that $v_{1}+\cdots+$ $v_{n} \equiv 0$ in this case.

We have seen in the derivation of the first equation in (2.4) that each solution $\psi \in$ $\mathcal{O}\left(x_{0}, t_{0}\right)$ of the equation $L \psi=\lambda \psi$ generates a column $\chi=\left(\chi_{1}, \ldots, \chi_{n}\right)^{T}$ satisfying the equation $\chi_{x}=(\Lambda(\lambda)+U(x, t)) \chi$ by the formulas $\chi_{1}=\psi, \chi_{2}=\psi^{\prime}, \ldots, \chi_{n}=\psi^{(n-1)}$, and vice versa. Likewise, the column $\eta=\left(\eta_{1}, \ldots, \eta_{n}\right)^{T}$ given by the formulas

$$
\begin{gathered}
\eta_{1}=\psi, \quad \eta_{2}=\left(\partial_{x}-v_{1}\right) \psi, \quad \eta_{3}=\left(\partial_{x}-v_{2}\right)\left(\partial_{x}-v_{1}\right) \psi, \quad \ldots, \\
\eta_{n}=\left(\partial_{x}-v_{n-1}\right) \cdots\left(\partial_{x}-v_{1}\right) \psi,
\end{gathered}
$$

satisfies the equation $\eta_{x}=(\Lambda(\lambda)+D(x, t)) \eta$, where $D(x, t):=\operatorname{diag}\left(v_{1}(x, t), \ldots, v_{n}(x, t)\right)$ is the diagonal matrix with entries $v_{1}, \ldots, v_{n}$. By multiplying out in formulas (2.6), one can linearly express the germs $\eta_{1}, \ldots, \eta_{n} \in \mathcal{O}\left(x_{0}, t_{0}\right)$ via $\chi_{1}, \ldots, \chi_{n}$ with coefficients depending on $v_{1}, \ldots, v_{n}$,

$$
\eta_{1}=\chi_{1}, \quad \eta_{2}=\chi_{2}-v_{1} \chi_{1}, \quad \eta_{3}=\chi_{3}-\left(v_{1}+v_{2}\right) \chi_{2}+\left(v_{1} v_{2}-v_{1}^{\prime}\right) \chi_{1}, \ldots .
$$

We obtain a lower triangular invertible $n \times n$ matrix $M(x, t)$ (with units on the diagonal) whose all entries are polynomials in the germs $v_{1}, \ldots, v_{n}$ and their $x$-derivatives such that the $n \times n$ matrix $Y(x, t, \lambda):=M(x, t) \Psi(x, t, \lambda)$ satisfies the equation $Y_{x}=(\Lambda(\lambda)+$ $D(x, t)) Y$. By differentiating formula $Y=M \Psi$ with respect to $t$ and by using the second equation in system (2.4), we conclude that

$$
Y_{x}=(\Lambda(\lambda)+D(x, t)) Y, \quad Y_{t}=S(x, t, \lambda) Y,
$$

where $S:=M_{t} M^{-1}+M R M^{-1}$ is a polynomial in $\lambda$ of the same degree as $R(x, t, \lambda)$.

At the final stage of the reduction process, we proceed to a basis in which the matrix $\Lambda(\lambda)$ is diagonal. To this end, it is convenient to introduce a new spectral parameter $z \in \mathbb{C} \backslash\{0\}$ by the formula $\lambda=z^{n}$. The eigenvalues of the matrix $\Lambda\left(z^{n}\right)$ have the form 
$z \alpha_{1}, \ldots, z \alpha_{n}$, where $\alpha_{1}, \ldots, \alpha_{n}$ are all $n$th roots of unity listed in arbitrary order, and the corresponding eigenvectors have the form $e_{j}=\left(1, z \alpha_{j}, \ldots,\left(z \alpha_{j}\right)^{n-1}\right)^{T}, 1 \leq j \leq n$. Thus, for all $z \in \mathbb{C} \backslash\{0\}$ one has $K(z)^{-1} \Lambda\left(z^{n}\right) K(z)=a z$, where $a:=\operatorname{diag}\left(\alpha_{1}, \ldots, \alpha_{n}\right)$ is the diagonal matrix with entries $\alpha_{1}, \ldots, \alpha_{n}$ and the columns of the matrix $K(z)$ are the above-mentioned eigenvectors; i.e., $K_{i j}(z):=\left(z \alpha_{j}\right)^{i-1}, 1 \leq i, j \leq n$. Note that $K(z)=\Delta(z) K(1)$, where $\Delta(z):=\operatorname{diag}\left(1, z, \ldots, z^{n-1}\right)$ is a diagonal matrix. Hence the matrix

$$
q(x, t):=K(z)^{-1} D(x, t) K(z)=K(1)^{-1} D(x, t) K(1)
$$

is independent of $z$. Since the $\operatorname{sum} \alpha_{1}^{l}+\cdots+\alpha_{n}^{l}$ is equal to $n$ for $l=0$ and is zero for all other $l$ in the interval $-n<l<n$, we can readily find the matrix entries $\left(K(1)^{-1}\right)_{i j}=$ $n^{-1} \alpha_{i}^{1-j}$ and $q_{i j}(x, t)=n^{-1} \sum_{k=1}^{n} v_{k}(x, t)\left(\alpha_{i}^{-1} \alpha_{j}\right)^{k-1}, 1 \leq i, j \leq n$. In particular, the property $v_{1}+\cdots+v_{n} \equiv 0$ implies that $q_{i i}(x, t) \equiv 0, i=1, \ldots, n$; i.e., the matrix $q(x, t)$ proves to be offdiagonal.

System (2.7) can be rewritten in terms of the new unknown $n \times n$ matrix $E(x, t, z):=$ $K(z)^{-1} Y\left(x, t, z^{n}\right)=K(z)^{-1} M(x, t) \Psi\left(x, t, z^{n}\right)$ in the form

$$
E_{x}=(a z+q(x, t)) E, \quad E_{t}=V(x, t, z) E,
$$

where so far we can only say that the $n \times n$ matrix $V(x, t, z):=K(z)^{-1} S\left(x, t, z^{n}\right) K(z)$ as a function of $z$ has the form $\sum_{k=-n}^{r+n} p_{k}(x, t) z^{k}$ with some matrices $p_{k}(x, t)$. (For example, negative powers of $z$ could in principle occur in this expression owing to the fact that $\left\{\Delta(z)^{-1} S\left(x, t, z^{n}\right) \Delta(z)\right\}_{i j}=z^{j-i} S_{i j}\left(x, t, z^{n}\right)$ for all $i, j=1, \ldots, n$.)

The following crucial lemma due to Drinfel'd and Sokolov says that $V(x, t, z)$ is actually a polynomial of degree $r$ in $z$ with leading coefficient $a^{r}$ and that the other coefficients of this polynomial can be expressed in a canonical way indicated in the lemma via the diagonal matrix $a$, the offdiagonal germ $q \in \mathcal{O}\left(x_{0}, t_{0}\right)$, and the coefficients $\gamma_{k}(t) \equiv \gamma_{k} \in \mathbb{C}$ in the representation (1.4) of the operator $P$. To state the lemma, let $\mathcal{R}\left(x_{0}\right)$ be the set of germs of all holomorphic gl $(n, \mathbb{C})$-valued mappings at an arbitrary point $x_{0} \in \mathbb{C}$, and let $\mathcal{R}_{\text {od }}\left(x_{0}\right)$ be the subset of all offdiagonal germs $\varkappa \in \mathcal{R}\left(x_{0}\right)$ (i.e., $\left.\varkappa_{i i}(x) \equiv 0, i=1, \ldots, n\right)$. Recall that a mapping $F: \mathcal{R}\left(x_{0}\right) \rightarrow \mathcal{R}\left(x_{0}\right)$ is called a differential polynomial if for each germ $\varkappa \in \mathcal{R}\left(x_{0}\right)$ every matrix entry of the germ $F(\varkappa)$ is a usual polynomial (the same for all $\varkappa$ ) of the matrix entries of $\varkappa$ and their $x$-derivatives. Next, it is convenient to extend the definition of the sequence $\gamma_{0}, \gamma_{1}, \ldots, \gamma_{r-2}$ of coefficients in (1.4) by setting $\gamma_{r-1}:=0$ and $\gamma_{j}:=0$ for all integer $j<0$.

Lemma 3. One has $p_{k}(x, t) \equiv 0$ for all integer $k<0$ and $k>r$ and $p_{k}(x, t)=$ $F_{r-k}(q)(x, t)$ for $k=0,1, \ldots, r$, where $F_{0}, F_{1}, F_{2}, \ldots$ is the unique sequence of differential polynomials with the following properties: $F_{0}(\varkappa) \equiv a^{r}, F_{m}(0) \equiv \gamma_{r-m} a^{r-m}, m=1,2, \ldots$, and the formal power series $\Phi(\varkappa, z):=\sum_{m=0}^{\infty} F_{m}(\varkappa) z^{-m}$ satisfies the differential equation $\partial_{x} \Phi(\varkappa, z)=[a z+\varkappa, \Phi(\varkappa, z)]$ identically in $\varkappa \in \mathcal{R}_{\mathrm{od}}\left(x_{0}\right)$ and in $z$. In particular, $p_{r}(x, t) \equiv a^{r}$.

Proof. Note that the existence and uniqueness of a sequence of differential polynomials with these properties are proved in [20. Lemma 1]; alternatively, they can be obtained from Proposition 1.2 and the description of the space $\Omega_{L}$ before formula (1.3) in 1 . The assertions of the Lemma themselves are equivalent to the formula for $\overline{\mathcal{A}}$ contained in the description of the modified Lax equations between Lemma 3.23 and Proposition 3.24 in [1] (Section 3.8, p. 117 of the Russian edition) in view of the notation in Section 1.1 and formula (2.3) in [1]. (The last formula essentially coincides with our formula (1.4).)

Remark 3. Let us illustrate the profoundness of the claim in Lemma 3 using the Boussinesq system (1.3) as an example. A straightforward computation on the basis of formula 
(2.4') Shows that the matrix $S$ in the second equation in system (2.7) has the form

$$
S(x, t, \lambda)=\left(\begin{array}{ccc}
w_{0}+v_{1}^{2}+v_{1}^{\prime} & v_{1}+v_{2} & 1 \\
\lambda-A & w_{0}-u_{1}-v_{1}\left(v_{1}+v_{2}\right) & -v_{1} \\
C & \lambda-B & w_{0}-u_{1}-v_{1}^{\prime}
\end{array}\right)
$$

in this case, where the expressions $A, B$, and $C$ are independent of $\lambda$ and can be expressed by the formulas

$$
\begin{aligned}
& A=v_{1 t}+u_{0}-w_{0}^{\prime}+v_{1}\left(u_{1}+v_{1}^{\prime}+v_{1}^{2}\right), \\
& B=\left(v_{1}+v_{2}\right)_{t}-\left(v_{1}+v_{2}\right)\left(v_{1} v_{2}-v_{1}^{\prime}\right)+u_{0}+u_{1}^{\prime}-2 w_{0}^{\prime}, \\
& C=\left(v_{1} v_{2}-v_{1}^{\prime}\right)_{t}-v_{1}\left(v_{1}+v_{2}\right)_{t}+w_{0}^{\prime \prime}-u_{0}^{\prime}+\left(v_{1}^{\prime}+v_{1}^{2}\right) v_{1} v_{2} .
\end{aligned}
$$

Part of claim of Lemma 3 (namely, the equations $p_{-2}(x, t) \equiv 0$ and $p_{-1}(x, t) \equiv 0$ ) is that in this case $A=B=C=0$ for all functions of the form

$$
v_{1}:=\frac{\psi_{1}^{\prime}}{\psi_{1}}, \quad v_{2}:=\frac{\left(\psi_{1} \psi_{2}^{\prime}-\psi_{1}^{\prime} \psi_{2}\right)^{\prime}}{\psi_{1} \psi_{2}^{\prime}-\psi_{1}^{\prime} \psi_{2}}-\frac{\psi_{1}^{\prime}}{\psi_{1}},
$$

where $\psi_{1}(x, t)$ and $\psi_{2}(x, t)$ are any solutions of the system $\psi^{\prime \prime \prime}+u_{1} \psi^{\prime}+u_{0} \psi=0, \psi_{t}=$ $\psi^{\prime \prime}+w_{0} \psi$ under the condition that the denominators of both fractions occurring in the formulas for $v_{1}$ and $v_{2}$ are nonzero at the point $\left(x_{0}, t_{0}\right)$. The reader will possibly agree that a straightforward verification of these identities would be at least cumbersome.

\section{$\S 3$. Proof of the Theorem}

(A) By Lemma 3, the auxiliary linear system (2.8) has the form

$$
E_{x}=U E, \quad E_{t}=V E,
$$

where $U=a z+q$ and $V=\sum_{k=0}^{r} F_{r-k}(q) z^{k}$ are polynomials in $z$ with matrix-valued coefficients depending on $x$ and $t$. If this system has a holomorphic solution $E: \Omega \rightarrow \operatorname{GL}(n, \mathbb{C})$ in some domain $\Omega \subset \mathbb{C}_{x, t}^{2}$ at least for one $z \in \mathbb{C}$, then the offdiagonal matrix function $q: \Omega \rightarrow \operatorname{gl}(n, \mathbb{C})$ determining the coefficients of the system satisfies the consistency condition $U_{t}-V_{x}+[U, V]=0$ (obtained by matching the second derivatives $E_{x t}=(U E)_{t}$ and $E_{t x}=(V E)_{x}$ and by cancelling out the invertible matrix $E$ ), which, as is well known (e.g., see [20, $\S ", 2]$ ), is independent of $z$ and can be represented in the form

$$
q_{t}=\left[a, F_{r+1}(q)\right] .
$$

Equations of the form (3.2) are called soliton equations of parabolic type in 21.

For each solution $u_{0}, u_{1}, \ldots, u_{n-2} \in \mathcal{O}\left(x_{0}, t_{0}\right)$ of system (1.5) equivalent to Eq. (1.1) for given $\gamma_{k}(t) \equiv \gamma_{k}=$ const in (1.4), we construct (see Section 2) a diagonal matrix $D(x, t)$ with entries in $\mathcal{O}\left(x_{0}, t_{0}\right)$ and a solution $E: \Omega \rightarrow \mathrm{GL}(n, \mathbb{C})$ of system (3.1) (for the offdiagonal matrix $\left.q(x, t)=K(1)^{-1} D(x, t) K(1)\right)$ in a small neighborhood $\Omega \subset \mathbb{C}_{x t}^{2}$ of the point $\left(x_{0}, t_{0}\right)$ for an arbitrary fixed value $z=z_{0} \in \mathbb{C} \backslash\{0\}$. For this construction, by Lemmas 1 and 2, it suffices (in an arbitrary way) to choose a matrix $\Psi_{0}(0) \in \operatorname{GL}(n, \mathbb{C})$ whose all principal minors are nonzero and a matrix $\Psi_{0}\left(\lambda_{0}\right) \in \operatorname{GL}(n, \mathbb{C})$, where $\lambda_{0}:=z_{0}^{n}$. Theorem $2(\mathrm{~B})$ in 4 states that the resulting holomorphic offdiagonal solution $q: \Omega \rightarrow \operatorname{gl}(n, \mathbb{C})$ of Eq. (3.2) can be analytically continued to a globally meromorphic function $x$ for each $t$ close to $t_{0}$ (in particular, for $t=t_{0}$ ). Then the same analytic continuation is possible for all entries $v_{1}(x, t), \ldots, v_{n}(x, t)$ of the diagonal matrix $D(x, t)=K(1) q(x, t) K(1)^{-1}$ and hence (owing to the expressions for $u_{0}, \ldots, u_{n-2}$ in the form of polynomials of $v_{1}, \ldots, v_{n}$ and their derivatives, obtained by multiplying out in (2.5) for the coefficients $u_{0}(x, t), \ldots, u_{n-2}(x, t)$ of the operator $L$. 
Remark 4. For Theorem 2(B) in [4] to apply, in it required (as is mentioned in [4] in the first sentence of the paragraph containing Eq. (6)) that the spectrum of the matrix $b=a^{r}$ (which is the leading coefficient of the polynomial $V(x, t, z)$ in system (3.1)) be simple, i.e., that its eigenvalues be distinct. Our matrix $a=\operatorname{diag}\left(\alpha_{1}, \ldots, \alpha_{n}\right)$ satisfies this condition if and only if $r$ is not divisible by $n$. Note that the theorem obviously fails for $r$ divisible by $n$. (For $P$ one can take an appropriate power of $L$.) Note also that the key point where the simplicity of the spectrum is used in the papers [4] and [20] is an application (e.g., to Eq. (5.3) in 20.) of the Sibuya theorem claiming that the formal solutions of singularly perturbed equations belong to certain Gevrey classes.

(B) As was noted in [4, Section 9(B)], in the situation considered the first equation $E_{x}=(a z+q(x, t)) E$ in system (3.1) has a fundamental solution system globally meromorphic in $x$ for each $t$ close to $t_{0}$ (including $t=t_{0}$ ) and for each $z \in \mathbb{C}$. Since the explanations given there are way too short, it is expedient to describe the proof of this assertion in more detail.

Clearly, it suffices to prove it for $t=t_{0}$. We assume that Eq. (3.2) has a holomorphic offdiagonal solution $q: \Omega \rightarrow \operatorname{gl}(n, \mathbb{C})$ in some neighborhood $\Omega \subset \mathbb{C}^{2}$ of the point $\left(x_{0}, t_{0}\right)$. By Theorem 2(A) in [4], it follows that the germ $q_{0}(x):=q\left(x, t_{0}\right)$ satisfies the condition $L q_{0} \in \mathrm{Gev}_{1 / r}$. Since we always assume that $r \geq 2$, so much the more we have $L q_{0} \in$ $\operatorname{Gev}_{1-0}$. Then the formal power series $f(z):=I+L q_{0}(z)$ and the Riemann problem $e^{a\left(x-x_{0}\right) z} f^{-1}(z)=\gamma_{-}^{-1}(x, z) \gamma_{+}(x, z)$ satisfy the assumptions of Theorem 3(B) in [4] for $m=1$. This theorem in particular says that the invertible matrix function $\gamma_{+}(x, z)$ (which is easily seen to have the same determinant as $e^{a\left(x-x_{0}\right) z}$ ) is defined for all $x \in \mathbb{C}$ except for the zero set of some entire function $\tau_{f}(x) \not \equiv 0$ and is a globally meromorphic function with denominator $\tau_{f}(x)$. However, the first equation in (18) in the proof of Lemma 12 in [4] (where $B \varphi=q_{0}$ by Lemma 12 itself) shows that the matrix $\gamma_{+}(x, z)$ can be viewed as a solution $E$ of the equation $E_{x}=\left(a z+q_{0}(x)\right) E$. Thus, we have found the desired globally meromorphic fundamental solution system.

Returning to the operator $\widetilde{L}=\partial_{x}^{n}+\sum_{j=0}^{n-2} \widetilde{u}_{j}(x) \partial_{x}^{j}$, note that for each $\lambda=z^{n} \in \mathbb{C} \backslash\{0\}$ and for all $x$ in a neighborhood of $x_{0}$ the ordinary differential equation $\widetilde{L} \varphi=\lambda \varphi$ has the fundamental solution system $\Psi\left(x, t_{0}, \lambda\right)=M\left(x, t_{0}\right)^{-1} K(z) E\left(x, t_{0}, z\right)$ (see the definition of $E(x, t, z)$ before formula (2.8) $)$, which, by the preceding (and also owing to the fact, mentioned before formula (2.7), that the $n \times n$ matrix $M\left(x, t_{0}\right)$ is lower-triangular with units on the diagonal and that all of its entries are polynomials of the functions $v_{1}\left(x, t_{0}\right), \ldots, v_{n}\left(x, t_{0}\right)$ and their $x$-derivatives $)$, can be extended to a globally meromorphic invertible matrix function of $x$. Thus, we have proved the trivial monodromy property of the operator $\widetilde{L}$ for all $\lambda \in \mathbb{C} \backslash\{0\}$.

The preceding argument fails for $\lambda=0$ (for example, the matrix $K(z)$ becomes noninvertible), but the trivial monodromy property itself remains valid, because it can be obtained from the case already considered by passing to the limit as $\lambda \rightarrow 0$. Indeed, let $B$ be the set of all poles of the coefficients of $\widetilde{L}$. It is discrete and at most countable. If $\varphi \in \mathcal{O}\left(x_{0}\right)$ is any solution of the equation $\widetilde{L} \varphi=0$ in a neighborhood of a point $x_{0} \in \mathbb{C} \backslash B$ and $\varphi(x, \lambda), 0<|\lambda|<1$, is the solution of the equation $\widetilde{L} \varphi=\lambda \varphi$ with the same initial data at $x_{0}$, then the functions $\varphi(x, \lambda)$ are globally meromorphic (this has already been proved), uniformly bounded on compact subsets of $\mathbb{C} \backslash B$ (by the Gronwall inequality), and converge to $\varphi(x)$ uniformly in a neighborhood of $x_{0}$ as $\lambda \rightarrow 0$ (by the theorem on the continuous dependence of solutions on a parameter). It follows by the compactness principle that the functions converge uniformly on compact subsets of $\mathbb{C} \backslash B$ and hence define an analytic continuation of the germ $\varphi(x)$ to a holomorphic function on $\mathbb{C} \backslash B$. Since all singular points $x_{s} \in B$ of the ordinary differential equation $\widetilde{L} \varphi=0$ are regular 
(which can be proved by applying the Fuchs regularity criterion, mentioned at the beginning of the proof of claim (C), to the equation $\widetilde{L} \varphi=\lambda \varphi$ twice, in one direction for any $\lambda \neq 0$ and in the opposite direction for $\lambda=0$ ), it follows that the possible singularities of $\varphi(x)$ at the points of $B$ can only be poles. Consequently, $\varphi(x)$ is meromorphic on $\mathbb{C}$, as desired.

(C) This property of poles of the coefficients of the operator $\widetilde{L}$ readily follows from the trivial monodromy property (B) (or even from the following weaker property: there exists a $\lambda \in \mathbb{C}$ for which all singular points of the equation $\widetilde{L} \varphi=\lambda \varphi$ are regular) by the classical Fuchs theorem. (See Section 15.3 of Ince's book 17] or the excellent modern exposition in [22, Theorem 4.2].)

However, one can also derive this property (and obtain some additional information in special cases; e.g., see Remark 6 below) directly (i.e., without explicitly using the Fuchs theorem) from formula (2.5) and the following lemma.

Lemma 4. All functions $v_{j}\left(x, t_{0}\right), 1 \leq j \leq n$, are the logarithmic derivatives of some globally meromorphic functions of $x$. In particular, all of their poles are of order one with integer residues.

Proof. We know from claim (B) that all entries of the matrix $\Psi\left(x, t_{0}, 0\right)$ extend to be globally meromorphic functions of $x$. Consequently, the same is true for the Wronskians $W_{j}(x), 1 \leq j \leq n$, defined in the statement of Lemma 2 and hence for the functions $f_{j}(x)=W_{j}(x) / W_{j-1}(x)$, whose logarithmic derivatives coincide with $v_{j}\left(x, t_{0}\right)$.

(D) The existence of a meromorphic continuation from the bidisk $D$ into the strip $S$ follows from Theorem 2(B) in 44 by the same argument as in (A) in the present proof. An example of a solution further continuable nowhere can be obtained from the corresponding example in [4] by formulas (2.5), and its noncontinuability is clear from the expressions of the functions $v_{j}$ via the Wronskians in Lemma 2 in view of claim (B) of our theorem.

(E) To each operator $L_{0}=\partial_{x}^{n}+\sum_{j=0}^{n-2} u_{j}(x) \partial_{x}^{j}$ with coefficients $u_{0}, u_{1}, \ldots, u_{n-2} \in$ $\mathcal{O}\left(x_{0}\right)$, the construction in Section 2 assigns a holomorphic offdiagonal gl $(n, \mathbb{C})$-valued function germ $q_{0}(x)$ at $x_{0}$. In this construction, we can take an arbitrary matrix $A \in$ $\operatorname{GL}(n, \mathbb{C})$ whose all principal minors are nonzero for the initial condition in Lemma 1 and an arbitrary ordering of the roots of unity in the matrix $a=\operatorname{diag}\left(\alpha_{1}, \ldots, \alpha_{n}\right)$. We denote the result produced by this construction by $q_{0}^{A, a}(x)$.

Then Theorem 2(A) in 4 and the argument in (A) in the present proof give the following criterion for the solvability of the local holomorphic Cauchy problem for Eq. (1.1), where $r, n \geq 2$ are arbitrary integers such that $r$ is not divisible by $n$ and $P$ is an arbitrary fixed operator of the form (1.4) with constant coefficients $\gamma_{k}(t)=\gamma_{k} \equiv$ const, $k=0,1, \ldots, r-2$. The Cauchy problem with the initial condition

$$
L=L_{0} \quad \text { for } \quad t=t_{0}
$$

for Eq. (1.1) has a local holomorphic solution of the form $L=\partial_{x}^{n}+\sum_{j=0}^{n-2} u_{j}(x, t) \partial_{x}^{j}$ with coefficients $u_{0}, u_{1}, \ldots, u_{n-2} \in \mathcal{O}\left(x_{0}, t_{0}\right)$ if and only if the condition $L q_{0}^{A, a} \in \mathrm{Gev}_{1 / r}$ holds for some (and hence for any) choice of the parameters $A$ and $a$. In this condition, the letter $L$ stands for the formal Laplace transform defined in [4] rather than for a solution of Eq. (1.1).

On the basis of this criterion, we say that an initial condition $L_{0}$ is a generic operator if there exist parameters $A$ and $a$ such that $L q_{0}^{A, a} \in \operatorname{Gev}_{\alpha}$ for some $\alpha<1 / r$. In this case, Theorem 2(C) in [4] guarantees that all coefficients $u_{0}, u_{1}, \ldots, u_{n-2} \in \mathcal{O}\left(x_{0}, t_{0}\right)$ of the solution $L$ of the above-mentioned Cauchy problem admit analytic continuation to 
globally meromorphic functions on the entire space $\mathbb{C}^{2}$. The proof of claim (E) and hence of the entire theorem is complete.

Remark 5. Although we do not claim that the set of generic operators is open or dense in any topology on the set of all operators, it seems intuitively to be a right thing to say that generic operators are, in a meaningful sense, a majority of all admissible initial conditions in the local holomorphic Cauchy problem for Eq. (1.1). Here the situation is completely similar to that for the heat equation. (Concerning the latter, e.g., see the survey [23] or Section 2 in [4.) By a well-known criterion (essentially due to Kovalevskaya but stated explicitly a quarter century after her work), the Cauchy problem

$$
\frac{\partial u}{\partial t}=\frac{\partial^{2} u}{\partial x^{2}}, \quad u\left(x, t_{0}\right)=u_{0}(x)
$$

has a local holomorphic solution $u \in \mathcal{O}\left(x_{0}, t_{0}\right)$ if and only if the germ $u_{0} \in \mathcal{O}\left(x_{0}\right)$ admits analytic continuation to an entire function of order $\leq 2$ and of finite type for order 2 . If $u_{0}(x)$ is an entire function of order $<2$ (this is an analog of generic initial conditions), then the solution $u(x, t)$ is an entire function on $\mathbb{C}^{2}$.

Returning to equations of Korteweg-de Vries type, note also that all of their finitegap solutions, as well as all solutions with converging Baker-Akhiezer function (see Remark 2), are generic solutions. For these solutions, one actually has $L q_{0}^{A, a} \in \mathrm{Gev}_{0}$ for an arbitrary choice of the parameters $A$ and $a$.

\section{$\S$ 4. Proof of the Corollary}

By changing the scale along the $x$ - and $t$-axes, one can assume that the coefficients $a$ and $b$ in Eq. (1.6) have the values $a=-\frac{1}{3}$ and $b=-\frac{4}{3}$. Every solution $u \in \mathcal{O}\left(x_{0}, t_{0}\right)$ of the resulting equation

$$
\frac{\partial^{2} u}{\partial t^{2}}=-\frac{1}{3} \frac{\partial^{4} u}{\partial x^{4}}-\frac{4}{3} \frac{\partial}{\partial x}\left(u \frac{\partial u}{\partial x}\right)
$$

generates a solution $u_{0}, u_{1} \in \mathcal{O}\left(x_{0}, t_{0}\right)$ (actually, even a one-parameter family of solutions) of the Boussinesq system (1.3) by the formulas

$$
u_{0}=\frac{1}{2}\left(\varphi+\frac{\partial u}{\partial x}\right), \quad u_{1}=u,
$$

where $\varphi \in \mathcal{O}\left(x_{0}, t_{0}\right)$ is an arbitrary solution of the system

$$
\frac{\partial \varphi}{\partial x}=\frac{\partial u}{\partial t}, \quad \frac{\partial \varphi}{\partial t}=-\frac{1}{3} \frac{\partial^{3} u}{\partial x^{3}}-\frac{4}{3} u \frac{\partial u}{\partial x},
$$

whose necessary and sufficient solvability condition is given by Eq. (4.1). Indeed, by substituting (4.2) into (1.3), we readily see that the first equation in (1.3) holds owing to the second equation in (4.3) and the relation $\varphi_{x x}=u_{t x}$, which follows from the first equation in (4.3), while the second equation in (1.3) is a straightforward consequence of the first equation in (4.3).

Now the desired claim on the continuability of $u(x, t)$ to a meromorphic function in $S$ follows directly from claim (D) of the theorem.

At this point, an example of a solution that is not continuable through any point of the boundary of $S$ can be given without resorting to the Boussinesq system. By the Cauchy-Kovalevskaya theorem, the Cauchy problem for Eq. (1.6) with initial conditions

$$
\partial_{x}^{k} u\left(x_{0}, t\right)=\varphi_{k}(t), \quad k=0,1,2,3,
$$

has a local holomorphic solution $u \in \mathcal{O}\left(x_{0}, t_{0}\right)$ for an arbitrary choice of the germs $\varphi_{k} \in \mathcal{O}\left(t_{0}\right), k=0,1,2,3$. For example, take a holomorphic function that is not meromorphically continuable through any boundary point of the disk $\left|t-t_{0}\right|<\delta_{2}$ for $\varphi_{0}(t)$ 
and identically zero functions for the other $\varphi_{k}(t)$. By applying the Cauchy-Kovalevskaya theorem to all points of the disk $\left\{\left(x_{0}, t\right) \in \mathbb{C}^{2}:\left|t-t_{0}\right|<\delta_{2}\right\}$, by the already proved part of the corollary we obtain a meromorphic solution $u(x, t)$ of Eq. (1.6) in the strip $S$. Should this solution admit a meromorphic continuation into a neighborhood of some boundary point $\left(x_{1}, t_{1}\right)$ of the domain $S,\left|t_{1}-t_{0}\right|=\delta_{2}$, it would follow from the already proved part of the corollary that this solution admits a meromorphic continuation into a neighborhood of the point $\left(x_{0}, t_{1}\right)$, and hence the function $\varphi_{0}(t)=u\left(x_{0}, t\right)$ would admit a meromorphic continuation into a neighborhood of $t_{1}$, which contradicts the choice of $\varphi_{0}$.

A completely similar combination of the already proved part of the corollary with the Cauchy-Kovalevskaya theorem shows that the envelope of meromorphy of an arbitrary germ $u \in \mathcal{O}\left(x_{0}, t_{0}\right)$ satisfying Eq. (1.6) has the form $\mathbb{C}_{x}^{1} \times X$, where $X$ is the envelope of meromorphy of the family of germs $\partial_{x}^{k} u\left(x_{0}, t\right) \in \mathcal{O}\left(t_{0}\right), k=0,1,2,3$, that is, the largest domain over $\mathbb{C}_{t}^{1}$ where all these four germs can be continued meromorphically (see [3, Section III.14.41]). On the other hand, every domain over $\mathbb{C}^{1}$ is a Stein manifold (e.g., see [24, Corollary 26.8 and the subsequent remark]) and hence the envelope of meromorphy of some family of four germs [3, Section III.14.41]. By taking these germs for the Cauchy data, we obtain a local solution with any prescribed envelope of meromorphy of the form $\mathbb{C}_{x}^{1} \times X$. This proves all claims of the corollary.

Remark 6. Let us indicate some other widely known nonlinear evolution equations for which all claims of the corollary hold. (Throughout the following, $a, b \in \mathbb{C} \backslash\{0\}, c \in \mathbb{C}$, and the subscripts indicate partial derivatives with respect to the corresponding variables.)

(A) The Korteweg-de Vries equation $u_{t}=a u_{x x x}+b u u_{x}$.

(B) The modified Korteweg-de Vries equation $u_{t}=a u_{x x x}+b u^{2} u_{x}$.

(C) The potential Korteweg-de Vries equation $u_{t}=a u_{x x x}+b u_{x}^{2}$.

(D) The nonlinear Schrödinger equation $u_{t}=a u_{x x}+b u|u|^{2}+c u$, where $|u|^{2}$ is by definition understood as $u(x, t) \overline{u(\bar{x}, \bar{t})}$. If we restrict ourselves to real values of the variables $(x, t) \in \mathbb{R}^{2}$ and choose the constants $a, b$, and $c$ appropriately, then we obtain the following versions of the nonlinear Schrödinger equation, which have straightforward physical meaning and are the main subject of study in the first part of the book [25]: the focusing NLS equation, the defocusing NLS equation, and the NLS equation with boundary conditions of finite density (see 25, Part I, Chapter I, Section 11, Remark 2]).

The claim in the corollary on the meromorphic continuation of an arbitrary solution from a bidisk into a strip can be obtained for equations (A), (B), and (D) by a straightforward application of Theorem 2(B) in [4. (The representation of these equations with appropriate $a, b$, and $c$ in the form (3.2) is well known and can be found, e.g., in (D) and $(\mathrm{E})$ at the end of Section 2 in $[20$.) Equation (C) cannot be represented in the form (3.2), but the derivative $v:=u_{x}$ of its arbitrary solution $u(x, t)$ holomorphic in the bidisk $D$ satisfies the equation $v_{t}=a v_{x x x}+2 b v v_{x}$ of the form (A) and admits meromorphic continuation into the strip $S$ by what was already proved. Furthermore, we readily find from Lemma 4 and formula (2.5) in the present paper that every solution of the Korteweg-de Vries equation (A) (which can always be reduced by scaling along the $x$ and $t$-axes to the form (1.2) with $\gamma_{1}(t) \equiv 0$ ) has only poles of second order with zero residue with respect to $x$. Thus, the antiderivative of this solution with respect to $x$ is globally meromorphic for each $t$ as well, and hence the original solution of equation (C) is meromorphic in $S$.

Now the second part (the last two paragraphs) of the proof of the corollary can be carried out without any modifications for all equations in question except for (D), where a slight generalization of the Cauchy-Kovalevskaya theorem (still covered by the standard proof using the majorant method) is needed. 


\section{REFERENCES}

[1] V. G. Drinfel'd and V. V. Sokolov, Lie algebras and equations of Korteweg-de Vries type, Current problems in mathematics, vol. 24, Itogi Nauki i Tekhniki, Akad. Nauk SSSR, Vsesoyuz. Inst. Nauchn. i Tekhn. Inform., Moscow, 1984, 81-180; English transl., J. Sov. Math. 30 (1985), 1975-2036. MR:760998 (86h:58071)

[2] G. B. Segal and G. Wilson, Loop groups and equations of KdV type, Publ. Math. IHES 61 (1985), 5-65. MR783348 (87b:58039)

[3] B. V. Shabat, Introduction to complex analysis. Part II: Functions of several variables, Nauka, Moscow, 1985; English transl., Transl. of Math. Monographs, vol. 110, Amer. Math. Soc., Providence, RI, 1992. MR1192135 (93g:32001)

[4] A. V. Domrin, Meromorphic extension of solutions of soliton equations, Izv. Ross. Akad. Nauk Ser. Mat. 74 (2010), no. 3, 23-44; English transl., Russian Acad. Sci. Izv. Math. 74 (2010), no. 3, 461-480. MR2682370 (2012a:37149)

[5] I. M. Krichever, Algebraic curves and commuting matricial differential operators, Funktsional. Anal. i Prilozhen. 10 (1976), no. 2, 75-76; English transl., Functional Anal. Appl. 10 (1976), no. 2, 144146. MR $0413179(54: 1298 \mathrm{~b})$

[6] I. M. Gelfand and L. A. Dikii, Fractional powers of operators and Hamiltonian systems, Funktsional. Anal. i Prilozhen. 10 (1976), no. 4, 13-29; English transl., Functional Anal. Appl. 10 (1976), no. 4, 259-273. MR0433508 (55:6484)

[7] L. A. Dickey, Soliton equations and Hamiltonian systems, 2nd ed., Advanced Series in Math. Phys., vol. 26, World Scientific, River Edge, NJ, 2003. MR 1964513 (2004c:37160)

[8] B. A. Dubrovin, I. M. Krichever, and S. P. Novikov, Integrable systems: I, Current problems in mathematics. Fundamental directions, vol. 4, Itogi Nauki i Tekhniki, Akad. Nauk SSSR, Vsesoyuz. Inst. Nauchn. i Tekhn. Inform., Moscow, 1985, 179-284; English transl., Dynamical systems IV, Symplectic geometry and its applications, Encycl. Math. Sci. 4 (1990), 173-280. MR842910 $(87 \mathrm{k}: 58112)$

[9] R. Beals, P. Deift, and C. Tomei Direct and inverse scattering on the line, Math. Surveys and Monographs, vol. 28, Amer. Math. Soc., Providence, RI, 1988. MR954382 (90a:58064)

[10] Yu. I. Manin, Algebraic aspects of nonlinear differential equations, Current problems in mathematics, vol. 11, Itogi Nauki i Tekhniki, Akad. Nauk SSSR, Vsesoyuz. Inst. Nauchn. i Tekhn. Inform., Moscow, 1978, 5-152; English transl., J. Sov. Math. 11 (1979), 1-122. MR0501136 (58:18567)

[11] F. Gesztesy, D. Race, K. Unterkofler, and R. Weikard, On Gelfand-Dickey and Drinfeld-Sokolov systems, Rev. Math. Phys. 6 (1994), no. 2, 227-276. MR1269299 (95g:58104)

[12] I. M. Krichever, Integration of nonlinear equations by the methods of algebraic geometry, Funktsional. Anal. i Prilozhen. 11 (1977), no. 1, 15-31; English transl., Functional Anal. Appl. 11 (1977), no. 1, 12-26. MR0494262 (58:13168)

[13] S. P. Novikov, A periodic problem for the Korteweg-de Vries equation: I, Funktsional. Anal. i Prilozhen. 8 (1974), no. 3, 54-66; English transl., Functional Anal. Appl. 8 (1974), no. 3, $236-246$. MR0382878 (52:3760)

[14] D. H. Sattinger and J. S. Szmigielski, Factorization and the dressing method for the Gelfand-Dikii hierarchy, Phys. D. 64 (1993), no. 1-3, 1-34. MR1214545 (94i:58096)

[15] A. V. Domrin, The Riemann problem and matrix-valued potentials with a converging BakerAkhiezer function, Teoret. Mat. Fiz. 144 (2005), no. 3, 453-471; English transl., Theor. Math. Phys. 144 (2005), 1264-1278. MR2191841 (2006g:30068)

[16] R. Weikard, On commuting differential operators, Electron. J. Differential Equations 19 (2000), 11 pp. MR.1744086(2001a:34146)

[17] E. L. Ince, Ordinary differential equations, Longmans, Green \& Co, London, 1927.

[18] A. P. Veselov and A. B. Shabat, A dressing chain and the spectral theory of the Schrödinger operator, Funktsional. Anal. i Prilozhen. 27 (1993), no. 2, 1-21; English transl., Functional Anal. Appl. 27 (1993), no. 2, 81-96. MR1251164 (94m:58179)

[19] G. Pólya and G. Szegö, Problems and theorems in analysis, vol. II, Springer, New York, 1976. MR.0396134(53:2)

[20] A. V. Domrin, Remarks on a local version of the method of the inverse scattering problem, Tr. Mat. Inst. Steklova 253 (2006), 46-60; English transl., Proc. Steklov Inst. Math. (2006), no. 2 (253), 37-50. MR2338686(2008k:37149)

[21] A. V. Domrin, The local holomorphic Cauchy problem for soliton equations of parabolic type, Dokl. Akad. Nauk 420 (2008), no. 1, 14-17; English transl., Dokl. Math. 77 (2008), no. 3, 332-335. MR2462096 (2009g:35251)

[22] A. A. Bolibrukh, Inverse monodromy problems in analytic theory of differential equations, MTsMNO, Moscow, 2009. (Russian) 
[23] G. S. Salekhov and V. R. Fridlender, On a problem inverse to the Cauchy-Kovalevskaya problem, Uspekhi Matem. Nauk (N.S.) 7 (1952), no. 5(51), 169-192. (Russian) MR0057430 (15:227c)

[24] O. Forster, Riemannsche Flächen, Heidelberger Taschenbücher, Bd. 184, Springer, Berlin etc., 1977; English transl., Lectures on Riemann surfaces, Graduate Texts in Mathematics, vol. 81. Springer, New York-Berlin, 1981. MR0447557 (56:5867)

[25] L. A. Takhtadzhyan and L. D. Faddeev, The Hamiltonian approach in soliton theory, Nauka, Moscow, 1986; English transl., Springer Series in Soviet Mathematics. Springer, Berlin, 1987. MR $889051(89 \mathrm{~m}: 58102)$

Faculty of Mechanics and Mathematics, Moscow State University, 1 Leninskie Gory, 119991 Moscow, Russian Federation

E-mail address: domrin@mi.ras.ru 